\title{
TEORIA DE LA VERDAD EN TARSKI
}

\author{
JUAN A. NUÑO \\ Universidad Central de Venezuela, \\ Caracas
}

Tarski es uno de los más conspicuos representantes de la corriente logicista dedicada al tratamiento semántico del concepto de verdad. Persistente también, pues allí donde otros investigadores en temas similares (Carnap, por ejemplo) dejaron hace tiempo de insistir en el esfuerzo por encuadrar lógicamente la vieja noción aristotélica de la correspondencia entre concepto y realidad (o entre proposición y estado de cosas; o entre signo y significado), Tarski ha seguido trabajando sobre el mismo problema, como lo revela la publicación de hace dos años en Scientific American del trabajo titulado "Truth and Proof". Se trata de la elaboración de unas conferencias dictadas en Berkeley en 1965 y en la Universidad de Londres en 1966. De esta forma, en total, Tarski abarca un período investigativo de casi cuarenta años sobre el tema de la verdad, pues fue en 1931 cuando produjo su primer artículo sobre la posibilidad de tratar semánticamente el concepto de verdad. Durante tan extenso lapso son tres las referencias específicas que permiten medir el aporte de Tarski en esta materia. Su primer y más largo trabajo llamado "El concepto de verdad en los lenguajes formalizados", aunque procede de 1931, fue publicado por primera vez en 1936; el ensayo titulado "La concepción semántica de la verdad y los fundamentos de la semántica" es de $1944 \mathrm{y}$, veinticinco años más tarde, en 1969, publica su artículo "Verdad y prueba".

De uno a otro trabajo hay variaciones en el tratamiento del problema, siendo más obvios los cambios en el último artículo que introduce notables diferencias respecto de los 
otros. No se trata tan sólo de registrar las transformaciones operadas en las posiciones de Tarski movidos de un simple interés historiográfico o erudito. Sucede que, con los cambios operados en los últimos trabajos, hay base suficiente para: 1) establecer los límites de la empresa semanticista original acerca del intento por lograr una definición de 'verdad'; 2) apreciar el esfuerzo por desplazar el problema de la verdad de su acepción filosófica tradicional a un terreno mensurable en principio matemáticamente.

La tesis original de Tarski asume una doble forma: $a$ ) en el contexto del lenguaje coloquial no es posible lograr una definición de verdad que resulte ser materialmente adecuada y formalmente correcta; $b$ ) el problema de lograr una definición de verdad alcanza una solución positiva en los lenguajes formalizados "pobres" o de orden finito (se entiende: de niveles lingüísticos), tal como el cálculo de clases, pero vuelve a resultar insoluble en el caso de lenguajes formalizados "ricos", de orden infinito, tal como la teoría general de las clases. La parte negativa de la tesis (esto es, el fracaso en obtener una definición de verdad tanto en el lenguaje coloquial como en los lenguajes formalizados de orden infinito) se explica a través de una forzosa elección metodológica (similar a la que se presenta en el caso de la medición de fotones según lo delimitado por el principio de indeterminación) : o se maneja el término 'verdad' (sus equivalentes adjetivos: 'verdadero', 'falso'), en cuyo caso se producen contradicciones; o se respetan las leyes lógicas (principio de tercio excluso y principio de no contradicción), en cuyo caso se debe renunciar al uso coherente de aquel término. En todo caso, la gran lección relativista que se desprende de las tesis centrales del primer trabajo de Tarski es que el término 'verdad' debe ser empleado y definido en un contexto lingüístico preciso, o como se dice textualmente:

we can only meaningfully say of an expression that it is 
true or not if we treat this expression as a part of a concrete language. ${ }^{1}$

Ello permite hacer un comentario interpretativo, destinado a utilizarlo como hilo conductor de los escritos posteriores. A saber: Tarski estableció una prohibición al mismo tiempo que acuñó un precepto. Al proceder así no se está insurgiendo contra el famoso dictum de Carnap, según el cual "no hay moral en la lógica", pues que hubiera moral no se entendió nunca como que no hubiera ninguna legalidad. Cierto que la frase de Carnap no es un dechado de claridad, ya que lo menos que necesitaría para una cabal comprensión, sería suministrar una definición de 'moral'. Podemos suponer entonces que Carnap entendía por 'moral' algo así como ley absoluta, en vista de que agregaba: "todo el mundo es libre de levantar su propia lógica", con tal de que (aquí venian las condiciones de este moralizante amoralista): $a$ ) se especifiquen claramente los métodos que se utilizan; $b$ ) se proporcionen reglas sintácticas del lenguaje construído. Justamente es lo que, por su parte, hizo Tarski. Prohibió por inútil cualquier intento de definir 'verdad' en el contexto del lenguaje coloquial y, para ello, se basó en el método de reducción al absurdo a través del análisis de la paradoja del mentiroso. Construyó, además, el precepto rela. tivista que obliga a situar el término 'verdad' en un contexto lingüístico bien determinado, para lo cual proporcionó reglas sintácticas, entre las que se destacan dos esenciales: i) todo lenguaje en el que se quiera calificar de 'verdaderas' o de 'falsas' a sus sentencias, debe poder distinguir entre nivelobjeto y nivel sobre lenguaje (metalenguaje); ii) no se po. drá incluir el metalenguaje en el nivel objeto, esto es, no será posible cerrar el lenguaje mediante una teoría general que, al versar sobre él mismo, lo circularice; es lo que, en su segundo gran escrito, llamará "lenguaje semánticamente cerrado". De no cumplir con el precepto, se reproducen las

1 Logic, Semantics, Metamathematics (Oxford, 1956), p. 263. 
antinomias que dan al traste con la pretendida legalidad ló. gica.

Con el tiempo, se ha mantenido en Tarski el precepto relativista y limitativo de los lenguajes formales no-contradictorios, pero se ha ido debilitando un tanto aquella prohibición sobre el lenguaje coloquial.

En efecto: ya en el primer escrito hay una vaga indicación de posible forma de tratamiento indirecto del lenguaje coloquial con el fin de poder emplear en él con éxito definitorio la noción de verdad. Al terminar su análisis probatorio de la imposibilidad de definir 'verdad' en lenguaje ordinario, Tarski entreabre una puerta por la que, más tarde, penetrará él mismo en la estructura del lenguaje colo. quial. Se trata del reconocimiento de la posibilidad de hacer extensivos al lenguaje coloquial los resultados alcanzables en lenguajes formalizados, pues como se señala:

if we translate into colloquial language any definition of a true sentence which has been constructed for some formalized language, we obtain a fragmentary definition of truth which embraces a wider or narrower category of sentences. $^{2}$

Semejante hipótesis de una partición de la verdad, mediante constitución fragmentaria de resultados definitorios, será ampliada en los dos escritos siguientes.

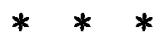

Doctrinariamente considerado, el segundo escrito de Tarski no se diferencia del primero. Se mantiene en él la doble tesis originalmente establecida. No hay posibilidad, por una parte, de definir 'verdad' en el lenguaje natural o en lo que, desde ahora, denominará Tarski lenguajes "semánticamente cerrados". Por otra parte, cualquier intento de definición debe venir precedido de una referencia al contexto lingüisti-

2 Op. cit. p. 165. 
co, y una vez localizado éste, acompañarse de una formalización mínima del lenguaje, que sirva para distinguir, al menos, entre 'lenguaje objeto' y 'metalenguaje'.

Se registran, no obstante, ciertos matices diferenciadores en el segundo escrito. Por ejemplo, en el rechazo al lenguaje cotidiano se ha suavizado en algo la posición demasiado excluyente y draconiana del primer escrito. Ahora ya no se le rechaza por producir contradicciones, sino que se le caracteriza como un lenguaje que no posee una estructura bien especificada, por lo cual no tiene sentido calificarlo de inconsistente o contradictorio. Se sitúa, por así decir, en una especie de limbo o coto aparte; ni es necesariamente semánticamente cerrado ni admite niveles metalingüísticos.

El cambio fundamental de este escrito se registra en lo referente a los términos definitorios empleados por Tarski para lograr, siempre en el marco de un lenguaje formalizado, una definición materialmente adecuada y formalmente correcta de 'verdad'. La solución se obtiene a través de lo que Tarski califica de "procedimiento indirecto" (o "desviado": roundabout way), que consiste en apoyarse en otra noción semántica, la de 'satisfacción', por ejemplo, para definir, por recursividad, la de 'verdad'. Reducido a su esquema más simple, el procedimiento propone considerar a toda sentencia como expresión de una función del tipo ' $x$ es $p$ ', la cual quedará satisfecha mediante la correcta sustitución de sus variables. Si se alcanza el caso límite en que la función sentencial es satisfecha por todas las posibles sustituciones válidas de sus variables, se podrá aseverar que la sentencia en cuestión es necesariamente verdadera.

El hecho de acudir a otro término del dominio semántico presenta, en principio, el problema lógico de un regressus ad infinitum o, cuando menos, enmascara una situación de sinonimia no justificada. Pues si para definir 'verdad' es menester acudir a 'satisfacción', a su vez, para definir 'satisfacción', será necesario echar mano de otro término (por ejemplo, 'denotación') y así sucesivamente. Parece evidente 
que, para evitar tales dificultades, Tarski tuvo que introducir, en este segundo escrito, una distinción entre, por una parte, 'verdad' y, por la otra, los restantes términos del dominio semántico. Semejante distinción lo que hace es crear una jerarquización interna de niveles en el empleo de los términos semánticos. Tarski sostiene por lo mismo que 'verdad' es un término "de naturaleza lógica diferente" a la de los restantes términos semánticos ('designación', 'definición', 'satisfacción', etc. esto es, los mismos que, en el primer escrito, habían sido calificados sin más de "análogos" a "verdad'). Y es de naturaleza lógica distinta porque mientras los otros términos son relaciones (entre ellos y entre los objetos por ellos referidos), el término 'verdad', dirá Tarski textualmente, "expresses a property (or denotes a class) of certain expressions, viz., of sentences".

Ante todo, ¿a qué obedece tal diferenciación? No sólo a evitar la dificultad técnica antes aludida, pues ello equivaldría a pensar que Tarski introduce la distinción ad hoc, sino que sirve para proporcionar un fundamento al intento de definición recursiva que se delínea ya en este texto, pero que se propondrá más específicamente en el tercero. En definitiva, lo que presenta Tarski es una definición extensional de 'verdad', según la cual 'verdad' es la clase de las sentencias verdaderas. Luego (razonamiento de Tarski), la naturaleza lógica de 'verdad' no se reduce a una simple relación (horizontal) entre términos y entidades, sino que establece una agrupación (vertical) entre clase y sus elementos.

Una diferenciación de este tipo conlleva ciertos problemas que o bien Tarski no ha visto, o bien ha preferido desdeñar. En primer lugar, es obvio que si 'verdad' expresa, denota una clase, por ello mismo, deja de ser un término semántico del nivel $l$ (relaciones entre términos y entidades) y pasa a ser un término semántico de nivel 2 o metasemántico (es decir, sintáctico), pues precisa de otros términos (semánticos) para poder operar. Dicho más brevemente: la condición semántica del término 'verdad' viene asegurada por la fun- 
ción semántica del término 'denotación' que aquella determina. Ello significa, por de pronto, que cualquier definición de 'verdad' ha de atender al complejo de relaciones entre un término ('verdad'), otro término semántico ('denotación') y el o los objetos denotados por aquel primero. Lo cual es algo muy distinto a definir una relación simple entre un término y el objeto a él referido. Sobreviene, además un problema mayor, del cual se resiente directamente la argumentación de Tarski a partir de este punto. Sucede que en la definición (mediante recursividad) de 'satisfacción' (de una función) se introduce, como apoyo lógico, el término en disputa: 'verdad'. Se cita directamnete a Tarski en este punto:

as regards the notion of satisfaction, we might try to define it by saying that given objects satisfy a given function if the latter becomes a true sentence when we replace in it free variables by names of given objects. ${ }^{3}$

Se crea, de esta manera, otra situación de circularidad. Si primero es caracterizada 'verdad' a través de 'denotación' (o su equivalente por categoría semántica, 'satisfacción'), en un segundo momento, se define a 'satisfacción' por medio de 'verdad'.

En resumen, respecto del primer escrito, la argumentación de Tarski, sin variar fundamentalmente su posición original, avanza a través de las precisiones de una técnica definitoria dirigida a encontrar una salida al problema mediante el recurso extensionalista. Aquella puerta entreabierta en el primer escrito con la referencia a una suma de definiciones frag. mentarias de verdad, se muestra ahora como el procedimiento recurisvo que podrá ser aplicado a conjuntos finitos de sentencias.

3 "The semantic conception of truth..." en, Readings in Philosophical Anaysis, H. Feigl-W. Sellars, eds. (New York, 1949), p. 63. 
En el tercero de los escritos de Tarski consagrados al tema de la verdad, es fácil observar cambios diversos respecto de los trabajos anteriores. Sólo para comodidad de agrupación, aquellos se clasificarán en cambios "menores" y "mayores".

Entre las modificaciones "menores", merecen destacarse tres, a saber: (i) la insistencia en el uso del término 'verdad'; (ii) la definición de la expresión 'materialmente adecuada' aplicada a la definición general de verdad; (iii) el tratamiento histórico-metodológico del valor de las antinomias del lenguaje natural.

El primero de estos cambios se registra en varios pasajes del escrito en cuestión: "la [explicación] que aquí se ofrece puede ser considerada en principio como una sugerencia para establecer un modo definitivo de empleo del término "verdadero', pero se acompaña de la creencia en la concordancia [agreement] con el uso prevaleciente de dicho término en el lenguaje cotidiano..." (subrayado nuestro). Es probable que ello obedezca a la influencia de las corrientes analíticas oxonianas y al segundo Wittgenstein. En cualquier caso, en este punto, Tarski adopta una línea media o de "compromiso" entre la posición normativa (que atiende al criterio de corrección) y la posición descriptiva (que se preocupa esencialmente por la frecuencia y modalidad del empleo). Prueba de su espíritu conciliatorio son las mismas líneas antes citadas.

Respecto de la aclaratoria de la expresión 'materialmente adecuada', que Tarski ha venido empleando como segunda nota caracterizadora de la definición de 'verdad' por él perseguida, es posible leer ahora: "el problema quedará resuelto por completo si logramos construir una definición general de verdad que sea adecuada en el sentido de que contenga, en tanto consecuencia lógica, todas las equivalencias de la forma (3)". Por su parte, la 'forma (3)' es la ya conocida de los primeros escritos, que reza: “ ' $p$ ' es verdadero si y sólo si $p$ ". Tal y como se venía entendiendo (aunque Tarski sólo lo especifica aquí por vez primera), una definición 
construida a partir de semejante fórmula será 'materialmente adecuada' cuando cubra todas las posibles equivalencias (por apropiada sustitución de variables) que admita la expresión funcional vacía. Reaparece, como se ve, el recurso extensionalista: la definición depende de las satisfacciones de las variables, siempre que se acepte que se está tratando con una clase no-vacía.

Por último, el background histórico introducido aquí por Tarski tiende a subrayar el valor metodológico de las antinomias: éstas sirven para probar un sistema lingüístico en la medida en que su aparición, por constituir un "sintoma de enfermedad", lo debilita lógicamente. Con ello, es obvio que sigue en pie la regla esencial que ha presidido, desde sus orígenes, todo el tratamiento tarskiano del problema de la verdad. Esto es: hay que elegir entre términos que, por su uso, producen contradicciones (expresables a través de antinomias) y sistemas debidamente formados que las excluyen.

También a tres pueden reducirse los cambios "mayores" que aporta el tercer escrito de Tarski sobre la verdad. Son, por orden creciente de importancia: (i) análisis de la tesis reduccionista, que tiende a eliminar del uso lingüístico lógico el término 'verdad'; (ii) suavización del concepto 'lenguaje formalizado' como dominio exclusiro del empleo no contradictorio de 'verdad'; (iii) presentación del mecanismo definitorio parcial de 'verdad' en combinación con la teoría de la prueba.

Lo que Kotarbinsky denomina "enfoque nihilista" del problema de la verdad es la posibilidad extrema contenida en la alternativa metodológica antes indicada: o se salvan las reglas de la lógica o se renuncia al empleo del término 'verdad'. Tarski recapitula lo esencial de semejante tesis. Lo que se prohibe con ella es el uso de 'verdad' en tanto predicado real que califique a nombres de sentencias. Queda reducido entonces su uso al de las expresiones adverbiales: 'es verdad que', 'no es verdad que', de hecho, absolutamente superfluas por su oblicuidad. Cualquier otro uso no sólo es 
innecesario por redundante, sino confundidor por carente de sentido. Tarski no acepta el expediente contenido en semejante tesis. Las dos razones que da para explicar su discon. formidad con la posición "nihilista" son reveladoras de su creciente ubicación en el dominio coloquial del lenguaje. Como primera razón, aporta un ejemplo (el del historiador de la ciencia que no podría utilizar la expresión 'todos los teoremas del autor $X$ han resultado ser verdaderos'), lo cual ya es suficientemente indicativo del respeto que Tarski ha adquirido por el uso del lenguaje cotidiano, pero no resulta ser suficientemente probatorio, pues cualquier defensor a ultranza de la tesis sino reduccionista, al menos, sustitutiva podría proponer como "traducción", que solucione el problema de la expresión aportada por Tarski, otra que dijera: 'todos los teoremas del autor $X$ han resultado ser válidos (o han sido probados) dentro del sistema $Y$ construido por dicho autor según las reglas $Z$ ' y entonces al autor Tarski, que ofrece una teoría de la prueba propia (véase más adelante), le resultaría muy difícil rechazar semejante lectura. La segunda razón presentada para oponerse a la tesis "nihilista" es de índole general y parece ser más bien una suerte de crítica cultural a la concepción reduccionista: "podría decirse que lo que hace la teoría nihilista de la verdad es eliminar la noción de verdad del acervo conceptual del espíritu humano [the conceptual stock of the human mind]". Sobre todo, la última expresión resulta extraña en un escrito de Tarski. Pareciera ser más propia de un neo-hegeliano. En todo caso, es obvio que el último Tarski está pendiente no sólo del "espíritu humano", sino del uso que, a través del lenguaje coloquial, haga aquél del término 'verdad'. Porque lo que viene a criticarle a la teoría nihilista es una supuesta hipocresía de conducta lingüística: aceptar por un lado lo que se llega a quitar por otro. Tal interpretación es la única forma posible de entender en toda su extensión la antes ci. tada expresión de Tarski: 
one could say that truth-theoretical "nihilism" pays lip service to some popular form of human speech, while actually removing the notion of truth from the conceptual stock of human mind."

Con independencia de cómo se entienda en este punto a Tarski, las dos referencias utilizadas, en la crítica al supuesto doble juego del "nihilismo" (esto es, las "formas populares del habla humana" y el "acervo conceptual del espíritu humano") aluden a contextos lingüísticos naturales, en modo alguno formalizados ni formalizables.

Ello conduce directamente al registro del segundo cambio mayor de Tarski en su último escrito sobre la teoría de la verdad. La oposición entre lenguaje coloquial y lenguajes formalizados no se presenta ni tan incompatible ni tan polarizada como en el primer escrito. En primer lugar, porque Tarski reserva metodológicamente los lenguajes formalizados para el dominio matemático, con una posible aplicación a las disciplinas empíricas. Pero, sobre todo, porque el lenguaje formalizado no es visto como "essentially opposed to natural language". Por el contrario, sostendrá Tarski: "los únicos lenguajes formalizados que parecen presentar un auténtico interés son aquellos que resultan ser fragmentos de los lenguajes naturales ...o aquellos que, al menos, pueden ser debidamente traducidos a lenguajes naturales". El cambio es radical respecto de las declaraciones anti-naturalistas del primer escrito. No es de descartar que en Tarski hayan influído, por un lado, los esfuerzos analistas por estudiar filosóficamente el lenguaje natural y, por otro, las limitaciones del formalismo que impiden alcanzar la formalización absoluta y obligan, en consecuencia, a una solución media entre lenguaje natural y símbolos formalizantes (lenguaje matemático del grupo Bourbaki, por ejemplo). Esa mayor receptividad de Tarski para el lenguaje coloquial no va a

4 "Truth and Proof", en Scientific American, June 1969, vol. 220, No. 6, p. 67). 
traducirse, sin embargo, en la posibilidad de establecer en él una definición general de 'verdad'. A todo lo más que llega Tarski es a admitir la posibilidad de formación de un esquema que funcione como definición general de 'verdad' $a$ partir de un fragmento de lenguaje natural que, por lo demás, se ha de ver sometido a ciertas condiciones.

A continuación, el plan de trabajo estipulado por Tarski. Tómese un fragmento de un lenguaje natural cualquiera. El lenguaje parcial $(L)$ así delimitado ha de cumplir con las siguientes tres condiciones: $a$ ) poseer reglas sintácticas lo suficientemente precisas como para permitir distinguir en él una sentencia de una expresión que no lo sea; $b$ ) poseer un número finito de sentencias; $c$ ) no figurar en él el término 'verdadero'. Ciertamente que la primera de tales condiciones ya determina algún grado de formalización, pues son precisamente las llamadas reglas de formación de un lenguaje las que permiten introducir la nota diferenciadora entre expresiones bien formadas y expresiones mal formadas y, dentro de aquellas, entre sentencias y no-sentencias. Por su parte, la tercera condición profundiza aún más esa tendencia a la formalización, ya que sirve para crear una distinción de niveles: si no se admite el uso de 'verdadero' en $L$, ello significa que va a emplearse desde otro lenguaje, es decir, que $L$ va a ser tomado como lenguaje-objeto y tratado en plan definitorio desde un metalenguaje. Pero es la segunda de esas condiciones la que hace que $L$ resulte ser un fragmento poco representativo de un lenguaje natural, ya que es caracteristico de los lenguajes cotidianos su posibilidad de agregar una nueva sentencia a la serie de las ya formadas, por lo que, en principio, todo lenguaje natural ha de ser considerado como contentivo de infinitas sentencias. La prueba es sencilla. Considérese un lenguaje natural $(S)$ como hipotéticamente finito (formado por $n$ sentencias). Al predicar de $S$ en el mismo $S$ que es un lenguaje finito formado por $n$ sentencias, se le agrega una nueva sentencia, con lo que pasa a ser $S$ contentivo de $n+1$ sentencias, en contradicción con la hipótesis. $Y$ 
así, al predicar de $S$ siempre en $S$ con $n+1$ sentencias que está formado por $n+1$ sentencias, se produce una serie infinita. QED.

Volviendo al modelo tarskiano. A partir de aquel lenguaje (parcial) semi-formalizado, Tarski sugiere que se tabulen las sentencias del mismo $\left(s_{1} ; s_{2}, s_{3}, \ldots s_{n}\right)$ y que para cada da una de tales sentencias (finitas) se construya una definición parcial de verdad mediante aplicación de aquellas al esquema que dice: " ' $p$ ' es verdadero si y sólo si $p$ ”, en donde ' $p$ ' se sustituye, en cada caso, por la sentencia en cues. tión. Se puede proceder luego a formar la suma lógica de todas las definiciones (parciales) así obtenidas, que tendría, en definitiva, la siguiente forma: "Para cada sentencia $x$ (de $L), x$ es verdadera si y sólo si o bien $s_{1}$, y $x$ es idéntica a ' $s_{1}$ ', o bien $s_{2}$, y $x$ es idéntica a ' $s_{2}$ ', o bien $\ldots s_{\mathrm{n}}$, y $x$ es idéntica a ' "." Piensa Tarski que, de esa manera, se alcanza una fórmula que puede ser considerada como "la deseada definición general de verdad: es formalmente correcta y es adecuada". El único problema que presenta es que o no puede aplicarse a la totalidad de un lenguaje natural $o$, de hacerlo, resultaría inoperante, aunque sólo sea por la infinidad de sentencias que contiene todo lenguaje ordinario, como antes se ha probado. Sólo en el caso de aceptar las limitaciones señaladas por Tarski, puede hablarse de solución de la definición de 'verdad' a nivel coloquial. Con ello, resulta manifiesto que Tarski sigue reservando el empleo de 'verdad' (y su intento de definición) para los lenguajes no coloquiales (o, al menos, no plenamente coloquiales). La diferencia respecto de las posiciones originales consiste en que, si en el primer escrito reservaba la posibilidad de definición y uso de 'verdad' a los lenguajes formalizados de orden finito (cálculo de clases), ahora intenta ampliar el dominio de aplicación a los que denomina "lenguajes científicos", en los cuales, a una parte de lenguaje natural (que opera en ellos como metalenguaje casi siempre), se le agrega otra de lenguaje formalizado, la cual hace el papel de proveedor de sen- 
tencias (finitas) y de reglas de formación.

Aparte de las críticas lingüísticas o contextuales (antes hechas) a la definición así obtenida por Tarski, sucede que la misma, desde un punto de vista metodológico, presenta un inconveniente práctico aun mayor: la definición permite decidir acerca del valor de verdad de cada sentencia sólo cuando ésta se sitúa en el contexto correspondiente a la disyunción o suma lógica del lenguaje al que pertenece, lo que significa que no se dispone de un criterio general que permita decidir operacionalmente acerca del valor de verdad de cada sentencia singularizada. Ello conduce a Tarski a la introducción del tercer cambio "mayor" en su último escrito. Se trata de lo que el propio Tarski denomina "procedimientos", que sirven para proporcionar "criterios parciales de verdad", específicos de cada ciencia o grupo de ciencias. Por tratarse, en este caso, de un tema lógico, situado por lo tanto en el grupo de ciencias deductivas, Tarski acude al procedimiento característico de tales ciencias: la teoría de la prueba.

Por más que ya en el segundo de los escritos aquí analizados, Tarski introdujera la relación entre 'verdad' y 'deducibilidad' (probability) de proposiciones (ver parágrafo 12 y nota 17 del citado escrito), es, sin embargo, en este tercero cuando extrae de tal relación una consecuencia importante para su teoría de la verdad. Se basa para ello en el hecho de la desigualdad existente en la relación entre "verdad' y 'prueba', pues como es sabido desde Gödel, ambas no son coextensas entre sí, lo que quiere decir que no hay coincidencia absoluta entre la clase de las proposiciones verdaderas y la clase de las proposiciones deducibles (provable). Del hecho (probado) que todas las proposiciones deducibles sean verdaderas, pero no todas las proposiciones verdaderas sean deducibles, Tarski postula la noción infinitista de 'verdad' entendida como límite de una serie: "la noción de proposición verdadera funciona así como el límite ideal que 
nunca puede alcanzarse, pero a cuya aproximación se tiende mediante una ampliación gradual del conjunto de proposiciones deducibles" (subrayado nuestro).

Al menos tres comentarios merece la teoría tarskiana de la verdad entendida como límite.

Ante todo, es evidente que Tarski sigue insistiendo en el recurso extensionalista para el tratamiento de la noción de verdad. 'Verdad' viene a ser, en consecuencia, conjunto (novacio, ampliable ad infinitum) de proposiciones verdaderas. Todas las observaciones que se hicieran antes siguen siendo ahora aplicables. Es asimismo manifiesto que Tarski está introduciendo, en forma poco precisa, una alteración al método axiomático. Cuando habla de "ampliación gradual" (by gradually widening the set... $)^{5}$ no puede referirse a otra cosa sino a la posibilidad de aumentar el número de axiomas de un sistema con el fin de lograr en él una mayor deducibilidad de proposiciones. Ahora bien, sabido es cuáles son los límites de tal recurso: o se debilitan los axiomas (por interdependencia) o se debilita el sistema al saturarlo (por inconsistencia).

Por último, al postular la noción de proposición verdade. ra como un "límite ideal nunca alcanzable", termina Tarski por manifestar abiertamente la equivocidad básica con la que, a lo largo de todos estos escritos, viene manejando el concepto de 'verdad'. Aquí hace referencia a la noción general, en tanto propiedad común a todas las proposiciones que la cumplen (esto es, 'verdad'='nombre de la clase de las proposiciones verdaderas'), pero en otros momentos, 'verdad' quedará reducida a la calificación de una proposición determinada ('verdad'='nombre de proposición'), pues cuando se trataba de construir el esquema disyuntivo de la definición de verdad, se partía de las adecuaciones parcia. les de cada proposición, considerada verdadera, que cum. pliera con ciertas condiciones. Esto es: cuando Tarski plantea el problema de definir 'proposición verdadera', lo puede

5 Op. cit., p. 76. 
hacer a un nivel metodológico relativo y, en tal caso, la definición que se intente coincidirá con el criterio específico que cada ciencia levante para calificar de 'verdadera' a sus sentencias. Pero cuando Tarski habla de la definición adecuada de 'verdad' (en los lenguajes naturales, aún fragmentarios), evade los límites metodológicos y se adentra en la zona platonizante de la esencia de la verdad, más allá de la designación relativa de 'verdadero', propia de cada senten. cia y limitada a un contexto previamente establecido. En el último de los escritos de Tarski, hay un pasaje en el que resulta bien patente la ambigüedad con que utiliza el término 'verdad', una vez en su dimensión universal y otra, limitándolo a cada objeto sentencial: "Puesto que la definición de verdad no proporciona semejante criterio [se refiere a criterios como los que suministran las definiciones de átomo o de gene o de vector] y, al mismo tiempo, se considera justamente la búsqueda de la verdad como la esencia de las actividades científicas, resulta un problema importante el encontrar al menos criterios parciales de verdad, y desarrollar procedimientos que permitan asertar o negar la verdad (o al menos, la semejanza de verdad) de tantas sentencias como sea posible."

Lo primero que se debería hacer es distinguir entre criterio parcial de verdad (aplicable a determinadas proposiciones, por ejemplo: tabla de valor) y búsqueda de la verdad, en tanto expresión vaga que denota la tendencia científica a un tipo de conocimiento objetivo y seguro. A partir de esa distinción, sólo puede aceptarse que cualquier intento defitorio de 'verdad' deberá convenir a cada conjunto de sentencias susceptibles de recibir el predicado 'verdadero'. De esta manera, la más elevada búsqueda de la verdad o definición general de verdad o elaboración de una teoría de la verdad, quedaría encuadrada en los no muy precisos límites de los caracteres propios del espíritu científico, sean ellos los que fueren. 
Alfred Tarski has dedicated three essays to the semantic treatment of the concept of truth. The first essay, "The Concept of Truth in Formalized Languages", was published in 1936, the second, "The Semantic Conception of Truth and the Foundations of Semantics" in 1944, and the last, entitled "Truth and Proof", in 1969. In each of these works there are changes in the way of dealing with the problem, and in the last one we find the most remarkable changes. An analysis of these changes gives sufficient reason: 1) to establish the limits of the original semantic task, intended to reach a definition of "truth", 2) to evaluate the effort in displacing the problem of truth from its traditional philosophical site to a field mathematically measurable. Tarski's original thesis has two aspects: a) in the context of ordinary language it is not possible to reach a definition of truth that is materially adequate and formally correct; b) it is possible to reach a definition of truth in finite formal languages, but not in infinite ones. We can notice through these three essays that Tarski bans every attempt to define "truth" in the context of ordinary language, and suggests the precept that the term "truth" must be placed within a well-determined linguistic context. Later, Tarski has maintained the precept, but has attenuated the restriction with respect to ordinary language.

The first essay indicates a possible way of treating ordinary language indirectly so as to allow the use of the notion of truth within it. The strategy would consist in extending to ordinary language the results achieved in formalized languages, by translating into it the definitions of true sentences obtained for some formalized languages.

In the second essay Tarski maintains the two aspects of the thesis originally established. Nevertheless, it is possible to note changes with respect to the definitory terms used to obtain a definition of truth in a formalized language. This method appeals to another semantic notion, that of 'satisfaction', to define recursively the notion of truth. Several remarks can be made concerning this procedure, for example the difficulty represented by the situation of circularity generated by the definitions of "truth" and "satisfaction".

In the third essay we can distinguish three important changes: i) The analysis of the reductionist thesis which tends to eliminate the term "truth" from its logical linguistic usage; ii) The broadening of the concept of "formalized language" considered as the exclusive 
domain of a non-contradictory use of "truth"; iii) The presentation of the partial definitory mechanism of "truth" combined with the theory of proof.

The reductionist thesis of the term "truth" is the extreme possibility contained in the methodological alternative: either saving the rules of logic, or giving up the employment of the term "truth" as a real predicate that qualifies the names of sentences. Tarski rejects this thesis and offers two reasons that reveal his increasing inclination towards the domain of ordinary language. The first reason is an example: if the nihilist thesis is accepted, it would be impossible to use the expression "all the theorems of the author $\mathrm{X}$ have turned out true". The second reason is more general: "it could be said that the nihilist theory of truth eliminates the notion of truth from the conceptual human stock". It is important to note that Tarski has in mind, ones more, ordinary linguistic contexts and not formalized ones.

The second important change consists in modifying the opposition between ordinary language and formalized languages. Tarski considers here that a formalized language is not essentially opposed to ordinary language. Nevertheless, this modification does not admit the possibility of establishing a general definition of "thruth" in ordinary language; Tarski only accepts the possibility of establishing a formal pattern based on a fragment of ordinary language that can function as a general definition of "truth". This pattern would estipulate three conditions for a fragment of any ordinary language: a) It must have syntactical rules precise enough to allow us to distinguish a sentence from an expression that is not a sentence; b) It must have a finite number of sentences; c) The term "true" must not appear in it. These three conditions make such a fragment scarcely representative of ordinary language, especially the second restriction. From this fragment of language Tarski suggests the tabulation of its sentences, and for each one he constructs a parcial definition of truth by applying to it the following pattern: "P is ture if and only if $\mathrm{p}$ ". Then he proceeds to form the logical sum of all definitions thus obtained. In this way we arrive at a formula that may be considered as a general definition of truth. Now, changing his original position. Tarski attempts to extend the definition of truth to scientific languages which are formed in part by ordinary language and in part by a formalized language. From a methodological point of view, this definition presents a practical problem: the definition allows us to decide the truth-value of every sentence only when it is placed in the context of the disjunction or logical sum of the language of which it is a part. This means that 
there is no general criterion which allows us to decide the truthvalue of an isolated sentence. This fact leads Tarski to the third change: the introduction of procedures to provide "partial criteria of truth", characteristic of each science or group of sciences. Since we are dealing in this case with logic, we appeal to the proper procedure, which is the theory of proof. We know that there is no perfect coincidence between the classs of true propositions and deducible propositions, since not every true proposition is deducible. From this, Tarski postulates the infinitist notion of "truth", understood as the limit of a series: the notion of a true proposition functions, then, as the ideal limit that cannot be reached, but to the approximation of which we tend, by means of a gradual extension of the class of deducible propositions.

Three remarks can be made on the theory of truth taken as a limit. First, it is evident that Tarski still insists on the extensionalist method of treating the notion of truth. Secondly, Tarski is introducing an alteration to the axiomatic method in a not very precise way. When he speaks of "gradual extension", he can refer to nothing but the possibility of increasing the number of axioms of a system, with the purpose of attaining a wider deducibility of sentences. The limit of this procedure is that either the axioms are weakened or the system is weakened when it is saturated. Finally, when Tarski postulates the notion of a true proposition as an "ideal limit that cannot be reached", he explicitly reveals, the basic ambiguity underlying his treatment of the notion of "truth" all through his essays. He refers to the general notion of truth as a common property of all propositions that are true; but some times, "truth" is reduced to the qualification of a definite proposition, since, when we were trying to construct the disjunctive pattern of the definition of truth, we started from the partial adequation to the pattern of each proposition considered as true and fulfilling certain conditions. So, in his last work, Tarski uses ambiguously the term "truth": at times it applies to the set of all true propositions and at others it is limited to each sentential object. Therefore, it is necessary to distinguish between the partial criterion of truth and the search for truth, understood as a vague expression that denots the scientific trend to reach a kind of objective and secure knowledge. 